\title{
Bifurcated SEN with Fluid Flow Conditioners
}

\author{
F. Rivera-Perez, ${ }^{1}$ C. Real-Ramirez, ${ }^{1}$ R. Miranda-Tello, ${ }^{2}$ R. Hernandez-Santoyo, ${ }^{1}$ \\ F. Cervantes-de la Torre, ${ }^{1}$ and J. Gonzalez-Trejo ${ }^{1}$ \\ ${ }^{1}$ Departamento de Sistemas, Division de Ciencias Basicas e Ingenieria, Universidad Autonoma Metropolitana-Azcapotzalco, \\ Avenida San Pablo 180, Col. Reynosa-Tamaulipas, Del. Azcapotzalco, 02200 Mexico, DF, Mexico \\ ${ }^{2}$ Departamento de Electronica, Division de Ciencias Basicas e Ingenieria, Universidad Autonoma Metropolitana-Azcapotzalco, \\ Avenida San Pablo 180, Col. Reynosa-Tamaulipas, Del. Azcapotzalco, 02200 Mexico, DF, Mexico
}

Correspondence should be addressed to J. Gonzalez-Trejo; jesus.glez.t@gmail.com

Received 29 November 2013; Accepted 15 April 2014; Published 30 June 2014

Academic Editor: Yonghong Wu

Copyright (C) 2014 F. Rivera-Perez et al. This is an open access article distributed under the Creative Commons Attribution License, which permits unrestricted use, distribution, and reproduction in any medium, provided the original work is properly cited.

\begin{abstract}
This work evaluates the performance of a novel design for a bifurcated submerged entry nozzle (SEN) used for the continuous casting of steel slabs. The proposed design incorporates fluid flow conditioners attached on SEN external wall. The fluid flow conditioners impose a pseudosymmetric pattern in the upper zone of the mold by inhibiting the fluid exchange between the zones created by conditioners. The performance of the SEN with fluid flow conditioners is analyzed through numerical simulations using the CFD technique. Numerical results were validated by means of physical simulations conducted on a scaled cold water model. Numerical and physical simulations confirmed that the performance of the proposed SEN is superior to a traditional one. Fluid flow conditioners reduce the liquid free surface fluctuations and minimize the occurrence of vortexes at the free surface.
\end{abstract}

\section{Introduction}

Increasing cleanliness of the steel slabs produced in continuous casting machines remains one of the priorities of the steel industry [1-3]. Ensuring product quality requires understanding a set of highly coupled phenomena. Several works have studied some of these phenomena separately $[1,4-9]$.

Researchers from academia and industry recognize that the fluid flow pattern in the mold significantly affects the quality of the steel produced in continuous casting machines [10-13]. However, the fluid flow pattern inside the mold in turn depends on several factors such as the configuration of the valve that connects the tundish with the submerged entry nozzle (SEN), the internal geometry of the SEN, the SEN submergence depth, the dimensions of the mold, and casting speed.

The many fluid flow patterns observed inside the mold could be grouped on three general types: double roll, unsteady, and single roll [14]. The preferred pattern is the double roll because it produces an equal distribution of molten slag layer over the entire width of the mold. In addition, the product displays the minimum number of steelmaking defects.

The design of the internal geometry of the SEN aims to induce a double roll fluid flow pattern inside the mold. Despite this, wrong operation conditions trigger a single roll or an unsteady fluid flow pattern. Some characteristics of an unsteady flow pattern are high mold level fluctuations, uneven molten slag layer thickness, and vortexing [14]. All these features significantly degrade the quality of the solidified steel (for instance, mold powder-based slivers, uneven solidification shell, and longitudinal cracks). The mechanism for transition from the double roll to an unsteady fluid flow pattern remains uncertain.

Several approaches have been used to damp fluctuations of the interface between molten slag and liquid steel in slab continuous casting. One of them is to impose an electromagnetic field across the mold to change the flow pattern $[15,16]$. This technology is the most commonly used, but it has several drawbacks associated with it; for example, it is sensitive to operating conditions, costly, and difficult to operate.

Immersing external refractory shapes in the mold is another approach recently proposed by Kamal and Sahai [17]. 
The authors claim that their device is a cheaper and more effective solution to the problem.

Following Kamal and Sahai, the present work proposes a new submerged entry nozzle design. The hypothesis behind this device is that fluid flow conditioners attached to the SEN external wall reduce the oscillations of the liquid surface. The flow conditioners impose a pseudosymmetric pattern in the upper zone of the mold by inhibiting the fluid exchange between the zones created by conditioners. The performance of the SEN with fluid flow conditioners is analyzed through CFD numerical simulations conducted on the commercial software ANSYS FLUENT [18]. The heat transfer and the solidification process at the mold section are beyond the scope of this work. However, the effect of the flow modifiers on both phenomena is briefly discussed. Numerical results were validated by means of physical simulations conducted on a scaled cold water model. This work shows that this novel design improves the performance of a traditional SEN.

\section{Description of the SEN with Fluid Flow Conditioners}

In theory, the design of the internal geometry of the SEN should produce a double roll pattern inside the mold under normal operation conditions. The former numerical simulations using the CFD technique supported this idea. For simplicity and computational limitations, 2D simulations covered only half of the mold. For the same reasons, the first 3D models used a quarter of the mold. This modeling approach forces symmetry restrictions on the numerical results. All these numerical simulations were done using a single phase, the liquid steel. The simulation of multiphase systems is relatively recent.

However, various defects in the cast steel suggested that the actual fluid flow pattern inside the mold is different from the ideal one. Physical simulations expose several phenomena contradicting the ideal pattern. These simulations used scaled models and water as the working fluid $[19,20]$.

The source of the phenomena that modify the ideal fluid flow pattern inside the continuous casting mold is still a subject of study. According to Real-Ramirez and GonzalezTrejo [10], the asymmetry of the flow pattern in the mold generates many of these phenomena. Figure 1 shows the zone close to the top of the mold, including a traditional bifurcated SEN. This figure also shows symmetry planes associated with $X$ and $Y$ axis, colored in blue and green, respectively. The symmetry planes divide the mold into four quarters. The following features of the fluid flow pattern inside the mold hold when the pattern is perfectly symmetrical.

(a) The imaginary symmetry plane colored in blue inhibits the fluid exchange between mold quarters (I) and (II), as well as the exchange between mold quarters (III) and (IV).

(b) The imaginary symmetry plane colored in green inhibits the fluid exchange between mold quarters (II) and (III), as well as the exchange between mold quarters (IV) and (I).

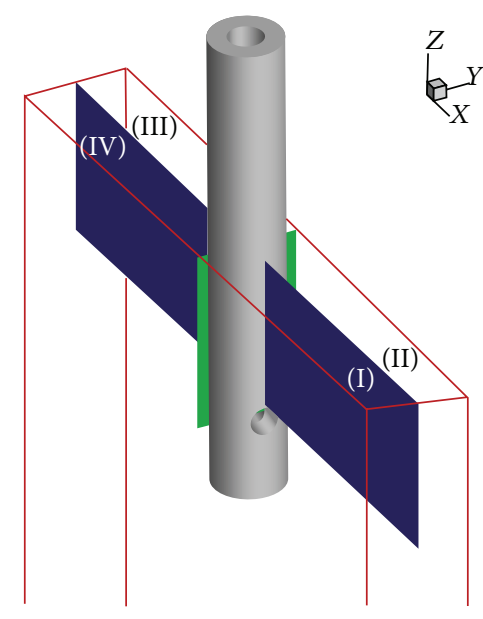

FIGURE 1: Upper zone of the mold. Symmetry planes define quarters (I), (II), (III), and (IV).

Asymmetry of the flow pattern allows fluid exchange between quarters in direct contact. This causes the formation of vortexes in the zone near to the liquid surface, which trap the molten slag that floats on top of the steel. Asymmetry also intensifies fluctuations of the liquid surface [19].

The SEN with fluid flow conditioners proposed in this work is depicted in Figure 2. This figure also shows the liquid (colored in blue) inside the mold. The SEN submergence depth is the height of the liquid measured from the center of the SEN outlet ports. The volume above the liquid surface is filled with air.

The aim of this design is to enhance the symmetry of the fluid flow pattern at the mold upper zone. To achieve this goal, we replace only one of the two imaginary symmetry planes by a physical barrier, named as fluid flow conditioner. A physical barrier on the other plane of symmetry could damage the formation of the solidified layer of steel in the central area of the wide walls of the mold. Our design is simple. Despite this, it has not been previously published nor patented.

This first design of the nozzle uses fluid flow conditioners of rectangular shape with constant cross section. The conditioner length and thickness values must be tuning to obtain optimal results. This analysis will be reported elsewhere. Subsequent works will report the performance of nozzles with fluid flow conditioners of trapezoidal shape with nonuniform cross section.

\section{Numerical Simulation of the SEN with Fluid Flow Conditioners}

3.1. Basic Mathematical Equations. If we consider that the flow is incompressible, we obtain the following Navier Stokes equation, which describes the fluid dynamics of a system:

$$
\rho\left(\frac{\partial \vec{v}}{\partial t}+\vec{v} \cdot \nabla \vec{v}\right)=-\nabla p+\eta \nabla^{2} \vec{v}+\rho f
$$

The numerical simulations used the standard $k-\varepsilon$ model for modeling turbulence. The turbulence kinetic energy, $K$, 


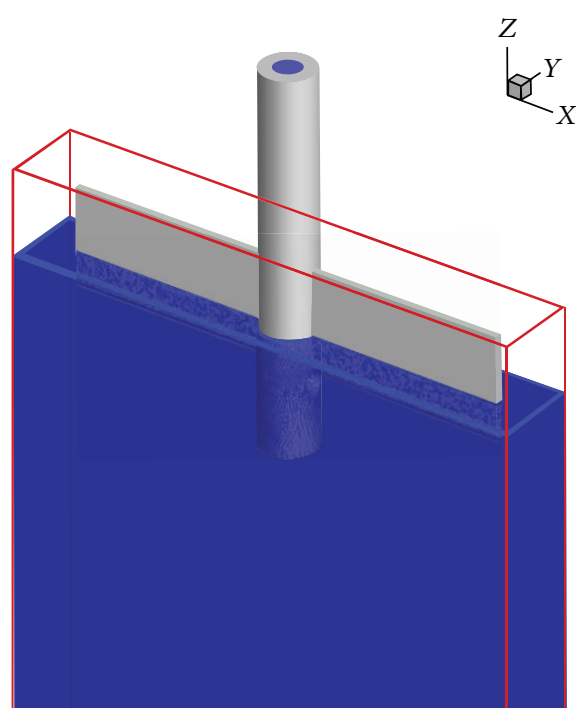

FIGURE 2: Sketch of the SEN with fluid flow conditioners.

and its rate of dissipation, $\varepsilon$, are obtained from the following transport equations:

$$
\begin{aligned}
& \frac{\partial}{\partial t}(\rho k)+\frac{\partial}{\partial x_{i}}\left(\rho k u_{i}\right) \\
& =\frac{\partial}{\partial x_{j}}\left[\left(\mu+\frac{\mu_{t}}{\sigma_{k}}\right) \frac{\partial k}{\partial x_{j}}\right]+G_{k}+G_{b}-\rho \varepsilon-Y_{M}+S_{k} \\
& \frac{\partial}{\partial t}(\rho \varepsilon)+\frac{\partial}{\partial x_{i}}\left(\rho \varepsilon u_{i}\right) \\
& =\frac{\partial}{\partial x_{j}}\left[\left(\mu+\frac{\mu_{t}}{\sigma_{\varepsilon}}\right) \frac{\partial \varepsilon}{\partial x_{j}}\right]+C_{1 \varepsilon} \frac{\varepsilon}{k}\left(G_{k} C_{3 \varepsilon} G_{b}\right) \\
& -C_{2 \varepsilon} \rho \frac{\varepsilon^{2}}{k}+S_{\varepsilon} .
\end{aligned}
$$

In these equations, $G_{k}$ represents the generation of turbulence kinetic energy due to the mean velocity gradients, $G_{b}$ is the generation of turbulence kinetic energy due to buoyancy, and $Y_{M}$ represents the contribution of the fluctuating dilatation in compressible turbulence to the overall dissipation rate. $C_{1 \varepsilon}, C_{2 \varepsilon}$, and $C_{3 \varepsilon}$ are constants. $\sigma_{k}$ and $\sigma_{\varepsilon}$ are the turbulent Prandtl numbers for $k$ and $\varepsilon$, respectively. $S_{k}$ and $S_{\varepsilon}$ are the user-defined source terms.

The model constants $C_{1 \varepsilon}, C_{2 \varepsilon}, C_{\mu}, \sigma_{k}$, and $\sigma_{\varepsilon}$ have the following default values [18]:

$$
\begin{gathered}
C_{1 \varepsilon}=1.440, \quad C_{2 \varepsilon}=1.920, \quad C_{\mu}=0.090, \\
\sigma_{k}=1.000, \quad \sigma_{\varepsilon}=1.300 .
\end{gathered}
$$

These values have been determined from experiments for fundamental turbulent flows. Several works have reported that these values are adequate for numerical simulations of the steel continuous casting.

3.2. Multiphase Model. The aim of the SEN with fluid flow conditioners is to inhibit the formation of vortexes in the zone near to the liquid surface, which trap the molten slag that floats on top of the steel. Another advantage of using flow conditioners is the damping of the fluctuations of the liquid surface. Therefore, numerical simulations include two fluids with different phase. The mathematical model has a liquid that enters into the mold through the SEN and a gas that floats over the liquid free surface inside the mold. The liquid used in numerical and physical simulations is water, in order to compare the results of them. For simplicity, the molten slag phase was omitted.

The Volume of Fluid (VOF) model is a surface-tracking technique applied to a fixed Eulerian mesh. It is designed for two immiscible fluids where the position of the interface between the fluids is of interest. In the VOF model, a single set of momentum equations is shared by the fluids, and the volume fraction of each of the fluids in every computational cell is tracked throughout the domain. In order to properly model multiphase flow, both spatial and time discretization schemes were used; therefore, a general transport equation can be written as

$$
\frac{\partial(\alpha \rho \phi)}{\partial t}+\nabla \cdot(\alpha \rho \vec{V} \phi)=\nabla \cdot \overline{\bar{\tau}}+S_{\phi}
$$

where $\phi$ is either a mixture or a phase variable, $\alpha$ is the phase volume fraction, $\rho$ is the mixture phase density, $\vec{V}$ is the mixture or phase velocity, $\overline{\bar{\tau}}$ is the diffusion term, and $S_{\phi}$ is the source term.

The tracking of the interface between the air and liquidwater phases is accomplished by the solution of a continuity equation for the volume fraction of one (or more) of the phases. For the qth phase, this equation has the following form:

$$
\frac{1}{\rho_{q}}\left[\frac{\partial}{\partial t}\left(\alpha_{q} \rho_{q}\right)+\nabla \cdot\left(\alpha_{q} \rho_{q} \vec{v}_{q}\right)=S_{\alpha_{q}}+\sum_{p=1}^{n}\left(\dot{m}_{p q}-\dot{m}_{p q}\right)\right],
$$

where $\dot{m}_{q p}$ is the mass transfer from phase $q$ to phase $p$ and $\dot{m}_{p q}$ is the mass transfer from phase $p$ to phase $q$; the source term $S_{\alpha_{q}}$ is equal to zero.

The volume fraction equation will not be solved for primary phase; the primary-phase volume fraction will be computed based on the following constraint:

$$
\sum_{q=1}^{n} \alpha_{q}=1
$$

To obtain the face fluxes for all cells, the implicit scheme is used for time discretization standard finite-difference 
interpolation schemes and the equation is written in the following form:

$$
\begin{aligned}
& \frac{\alpha_{q}^{n+1} \rho_{q}^{n+1}-\alpha_{q}^{n} \rho_{q}^{n}}{\Delta t} \\
& \quad+\sum_{f}\left(\rho_{q}^{n+1} U_{f}^{n+1} \alpha_{q, f}^{n+1}\right)=\left[S_{\alpha_{q}}+\sum_{p=1}^{n}\left(\dot{m}_{p q}-\dot{m}_{q p}\right)\right] V .
\end{aligned}
$$

Since this equation requires the volume fraction values at the current time step, a standard scalar transport equation is solved iteratively for the secondary-phase volume fraction (air) at each time step.

A single momentum equation is solved throughout the domain, and the resulting velocity field is shared among the phases. The momentum equation is dependent on the volume fractions of all phases through the properties $\rho$ and $\mu$ :

$$
\frac{\partial}{\partial t}(\rho \vec{v})+\nabla \cdot(\rho \vec{v} \vec{v})=-\nabla p+\nabla \cdot\left[\mu\left(\nabla \vec{v}+\nabla \vec{v}^{T}\right)\right]+\rho \vec{g}+\vec{F} .
$$

For time-dependent VOF calculations, (2) is solved using an explicit time-marching scheme.

The effects of surface tension along the interface between the air and liquid water phases are included. The VOF model can be augmented by the additional specification of the contact angles between the phases. The value of the surface tension coefficient was constantly equal to 1.400 .

These flows involve the existence of a free surface between the flowing fluid and air phase above it, acting as the atmosphere. In such cases, wave propagation and free surface behavior become important. Flow is governed by the forces of gravity and inertia. Open channel flows are characterized by the dimensionless Froude Number, which is defined as the ratio of inertia force and hydrostatic force:

$$
\mathrm{Fr}=\frac{V}{\sqrt{g y}},
$$

where $V$ is the velocity magnitude, $g$ is gravity, and $y$ is a length scale, which is the distance from the bottom of the mold to the free surface and is defined as constant initial boundary condition equal to 1.150 . When $\mathrm{Fr}<1.000$, which is the case, the flow is known to be subcritical where disturbances can travel upstream as well as downstream.

3.3. Geometrical Dimensions and Boundary Conditions. Numerical results of the mathematical model described above will be validated with a scaled model and using cold water as working fluid. Therefore, the geometrical dimensions and boundary conditions of the mathematical model coincide with that of the scaled model, which is a rectangular prism made of acrylic plates (see Figure 3(a)). It has a $1: 1 / 3$ scale ratio and its dimensions are $1.200 \mathrm{~m}$ length, $0.500 \mathrm{~m}$ width, and $0.070 \mathrm{~m}$ thickness. The water is discharged into the mold by the SEN, which is a cylindrical bifurcated pipe. The SEN is at the center of the mold, in the upper zone.

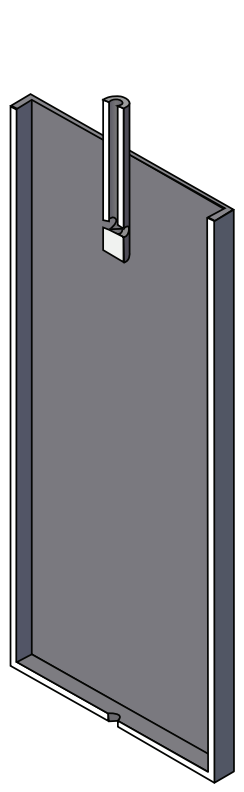

(a)

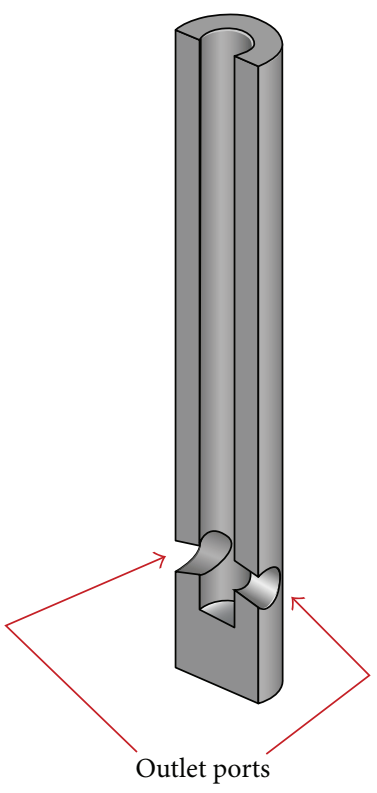

(b)

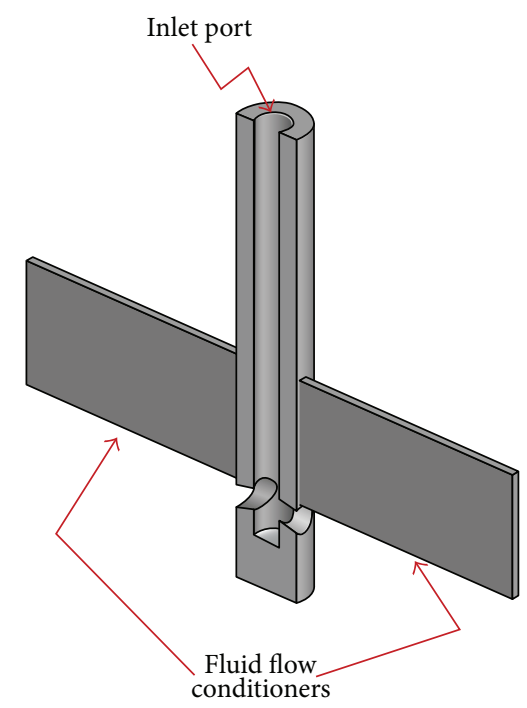

(c)

FIGURE 3: Sketch of the physical model. (a) Isometric view of a half of the mold. (b) Inset showing the inner geometry of a traditional SEN. (c) Inset showing the inner geometry of the SEN with conditioners.

The dimensions of the SEN are $0.320 \mathrm{~m}$ of length, $0.054 \mathrm{~m}$ of external diameter, and $0.0254 \mathrm{~m}$ of inner diameter. The SEN has two exit ports with a diameter of $0.020 \mathrm{~m}$. Each port has an inclination of 15 degrees downward with respect to the horizontal (see Figures 3(b) and 3(c)). The SEN is at the mold centerline. Each fluid flow conditioner is an acrylic plate of $0.175 \mathrm{~m}$ width, $0.090 \mathrm{~m}$ length, and $0.006 \mathrm{~m}$ thickness. The SEN submergence depth is equal to $0.050 \mathrm{~m}$, measured from the liquid free surface.

The primary inlet boundary condition is the liquid inlet velocity. Water enters into the mold through the inlet port at the top of the nozzle. The liquid velocity has a constant value 

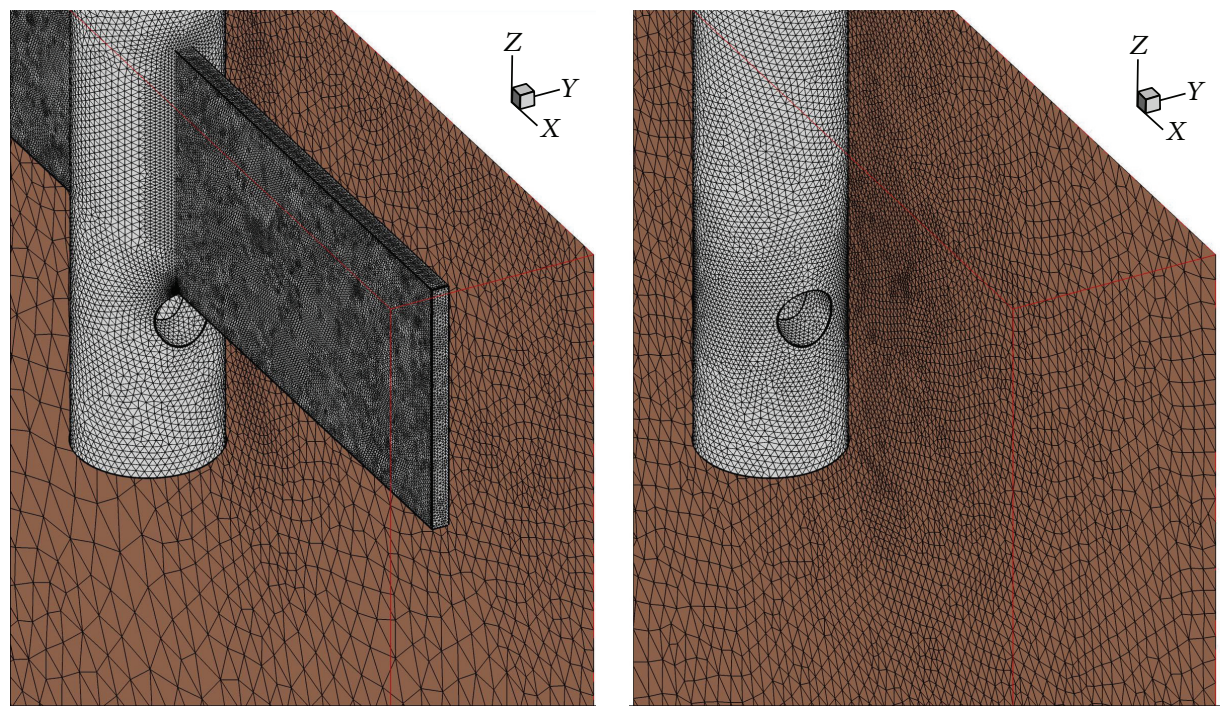

FIGURE 4: Meshing on the SEN external wall and the mold wide wall. The figure includes the SEN with fluid flow conditioners and a traditional SEN.

of $1.150 \mathrm{~m} / \mathrm{s}$. This value corresponds to a nominal casting speed of $1.800 \mathrm{~m} / \mathrm{min}$.

The secondary boundary condition is the pressure inlet condition, defined at the upper wall of the mold. At this boundary, the pressure has a constant value of $101325 \mathrm{~Pa}$. The total pressure $p_{0}$ at the inlet can be given as

$$
p_{0}=\frac{1}{2}\left(\rho-\rho_{0}\right) V^{2}+\left(\rho-\rho_{0}\right)|\vec{g}|(\hat{g} \cdot(\vec{b}-\vec{a})),
$$

where $\vec{b}$ and $\vec{a}$ are the position vectors of the face centroid and any point on the free surface, respectively. $\vec{g}$ is the gravity magnitude, $\hat{g}$ is the unit vector of gravity, $V$ is the velocity magnitude, $\rho$ is the density of the mixture in the cell, and $\rho_{0}$ is the reference density.

The third boundary condition corresponds to the outlet at the bottom of the mold. This wall is as an outflow boundary. The flow rate weighting value is 1.000 .

3.4. Discretization of Numerical Models. The models representing the mold with a traditional SEN and the model using the SEN with fluid flow conditioners were discretized employing similar criteria. The size of the minimum element was $1.000 \times 10^{-5} \mathrm{~m}$. Pyramidal elements grow linearly with a constant value of 1.050. Curvature and distance criteria were included. Therefore, models have a bigger elements density in the vicinity of the nozzle and near the liquid free surface.

For illustrative purposes, exclusively, Figure 4 shows the mesh on the SEN external wall and the wide wall of the mold. This figure includes a traditional SEN and the SEN with fluid flow conditioners.

3.5. Results and Discussion. In this section, we present the results of transient numerical simulations using the models, boundary conditions and operating conditions as described in the previous section. The transient numerical simulations represent 60 seconds of operation of the physical model. It was observed that this process is time enough to eliminate the behaviors associated with the start of the simulation. It was also observed that both models reached a quasi-steady state using 60 seconds of operation time. However, fluctuations in the free liquid surface are higher for the mold with the traditional nozzle.

Figure 5 shows the numerical reconstruction of the free liquid surface. This figure includes the results of the nozzle with fluid flow conditioners and the traditional nozzle. The shape of the liquid free surface for the traditional SEN is similar to that obtained in physical simulations [20]. This figure clearly shows that the SEN with conditioners produces a free surface with less distortion. In fact, the free surface remains almost flat a considerable portion of the mold width using a SEN with flow conditioners. In the zone close to the traditional SEN, there is a depression (circled in green), which is characteristic of the formation of vortexes.

Figure 6 shows the fluid velocity field on a plane parallel to the mold wide walls using the SEN with flow conditioners and a traditional SEN. All the vectors have the same length, but they are colored in accordance to its velocity magnitude. The plane is located close to one-mold-wide wall and below the free surface. The fluid flow pattern formed on the sample plane shows that the roll zone below the free surface has less distortions using the nozzle with fluid flow conditioners. Moreover, it is observed that the traditional nozzle creates an unexpected recirculation zone (circled with a red dashed line). This zone is located below the free surface near one of the broad walls and almost half of the distance between the thin wall and the nozzle. The figure belonging the traditional SEN shows a recirculation zone highlighted with a green solid line. This zone is close to the SEN.

Figure 7 shows the liquid velocity vectors on the free surface. This figure shows the results of both, the SEN with flow conditioners and a traditional SEN. All the liquid velocity 

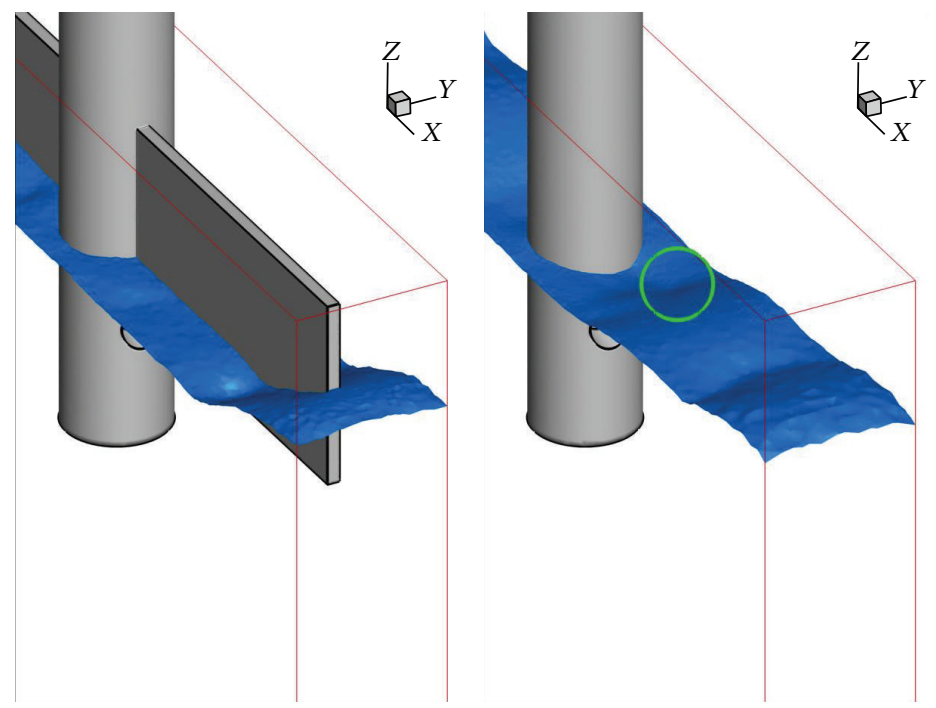

FIGURE 5: Numerical simulation of the free liquid surface on the mold using the SEN with flow conditioners and a traditional SEN.
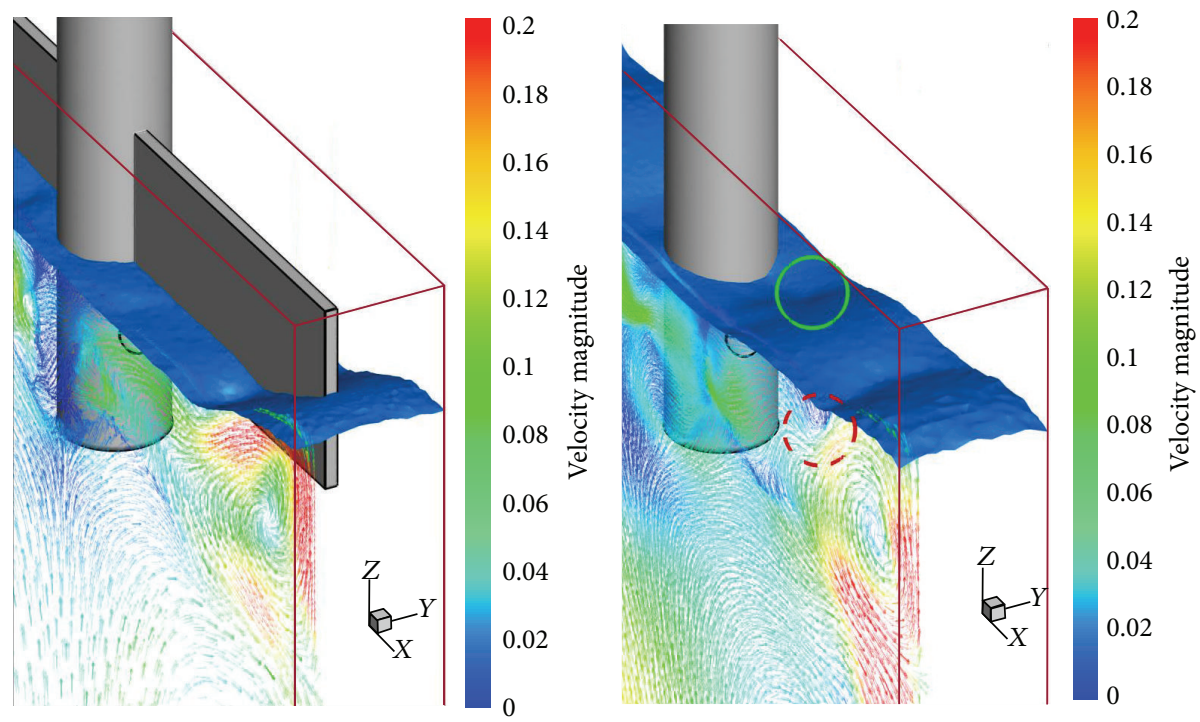

FIGURE 6: Fluid velocity field on a plane parallel to the mold wide walls using the SEN with flow conditioners and a traditional SEN. The plane is located close to one-mold-wide wall.

vectors have the same length. This figure highlights the same two recirculation zones described in Figure 6. Additionally, this figure clearly shows the zones in which fluid from quarter (I) cross to the quarter (II) when a traditional SEN is employed (zone circled with a yellow dot-dashed line).

The aim of the SEN fluid flow conditioners is to induce at the top of the mold a fluid flow pattern as symmetric as possible. Figure 8 shows contours plots of $y$-velocity on a plane at $z=1.100 \mathrm{~m}$ since the bottom of the mold. This plane is perpendicular to the mold wide walls and is located between the liquid free surface and the SEN outlet ports. In this figure, a positive $y$-velocity value means that $y$-component of the velocity vector is pointing from the left hand side of the reader to the right side. A negative value of the $y$-velocity means that the $y$-component of the velocity is pointing in the opposite direction. Figure 8 shows wide zones close to mold center with extreme values (too high and too low) when using a traditional SEN. In some of those zones, the fluid belonging to a certain mold quarter crosses to other quarter (see Figure 1). In contrast, the $y$-velocity values at almost all the plane remain close to zero when using a SEN with fluid flow conditioners.

Figure 9 shows the fluid velocity vectors at the same plane studied in Figure 8. This figure helps to visualize with clarity the way the fluid behaves in that zone. All the liquid velocity vectors have the same length. The figure on the left-hand side shows that the conditioner indeed directs the flow coming from the narrow wall to the center of the mold. In the figure belonging to the results of a traditional SEN, the zones where fluid from quarter (II) cross to the quarter (I) were also circled 

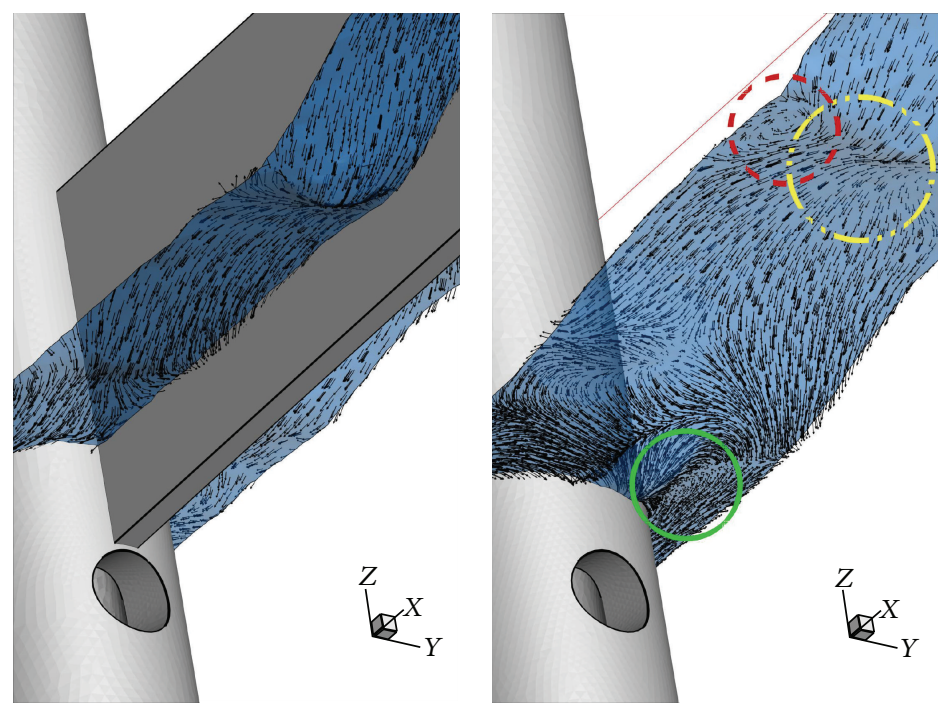

FIGURE 7: Liquid velocity vectors on the free surface. The figure shows the results of both the SEN with flow conditioners and a traditional SEN.
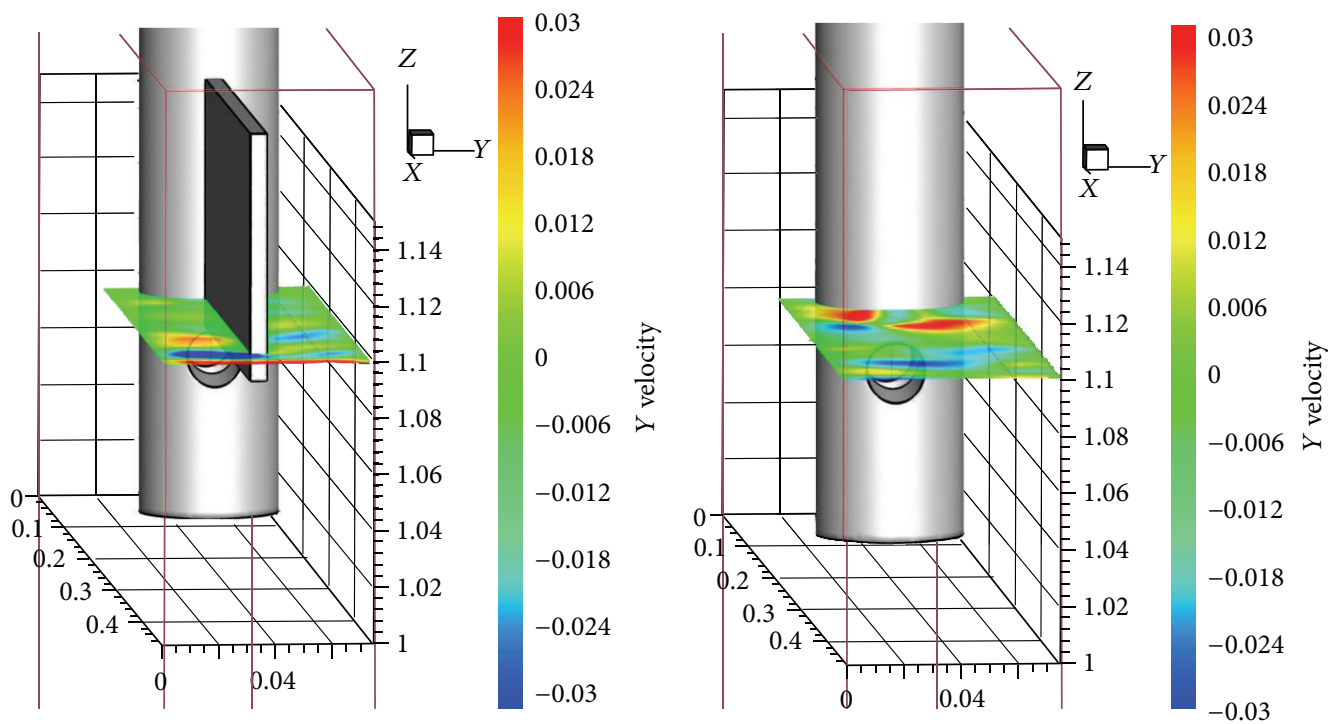

FiguRE 8: $Y$-velocity contours on a plane located at $z=1.1 \mathrm{~m}$.

with a yellow dashed line. Recirculation zones were circled with red solid lines.

Results previously presented and discussed have shown qualitatively that the fluid flow conditioners indeed improve the behavior of the SEN. Table 1 summarizes the results of an analysis of the fluid flow pattern inside a liquid volume near the liquid free surface. This analysis allows us to evaluate quantitatively the performance of the SEN with fluid flow conditioners. The boundaries of the control volume are $0.264 \mathrm{~m} \leq x \leq 0.436 \mathrm{~m} ; 0.00 \mathrm{~m} \leq y \leq 0.075 \mathrm{~m} ; z \geq 1.090 \mathrm{~m}$. Table 1 reports the average values of the $y$-velocity, $x$-velocity, and the velocity magnitude over the control volume for a traditional SEN and the SEN with fluid flow conditioners. This table confirms the comments that arose from Figures 8
TABLE 1: Analysis of the fluid flow pattern inside a volume near the liquid free surface.

\begin{tabular}{lcc}
\hline \multirow{2}{*}{ Variable } & \multicolumn{2}{c}{ Average values over the control volume } \\
& Traditional SEN & SEN with flow conditioners \\
\hline$y$-velocity & $-2.041289 \times 10^{-3}$ & $8.948284 \times 10^{-5}$ \\
$x$-velocity & $-4.936211 \times 10^{-2}$ & $-8.935193 \times 10^{-2}$ \\
Velocity magnitude & $6.853202 \times 10^{-2}$ & $1.006960 \times 10^{-1}$ \\
\hline
\end{tabular}

and 9. The average $y$-velocity for the traditional SEN is 22 times bigger than that observed when using a SEN with fluid flow conditioners. 

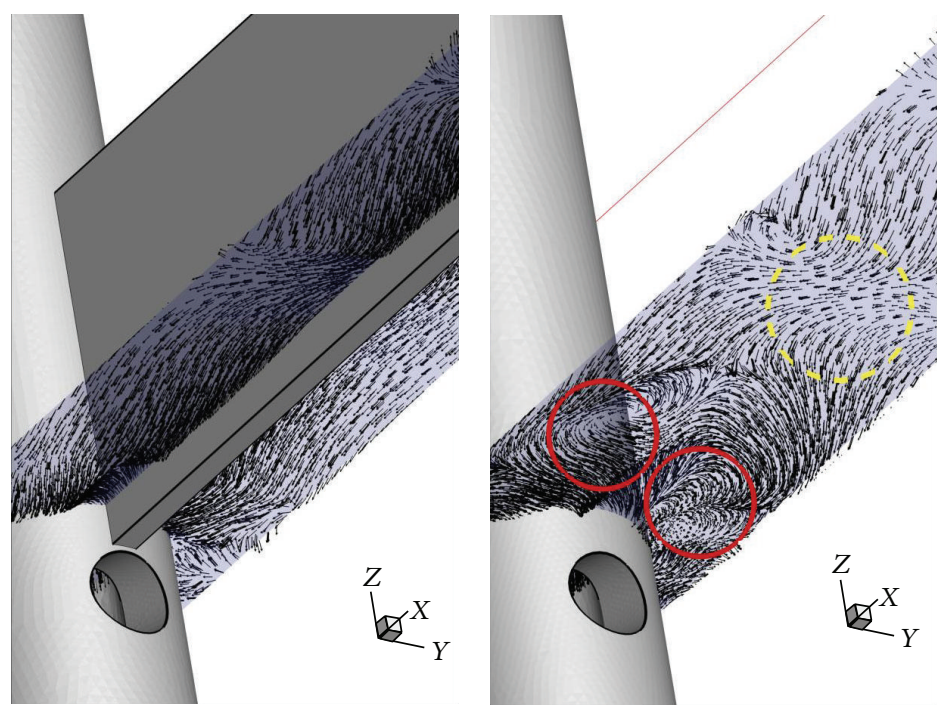

FIGURE 9: Liquid velocity vectors on a plane perpendicular to the mold wide walls. The plane is located between the liquid free surface and the SEN outlet ports. The figure shows the results of both the SEN with flow conditioners and a traditional SEN.
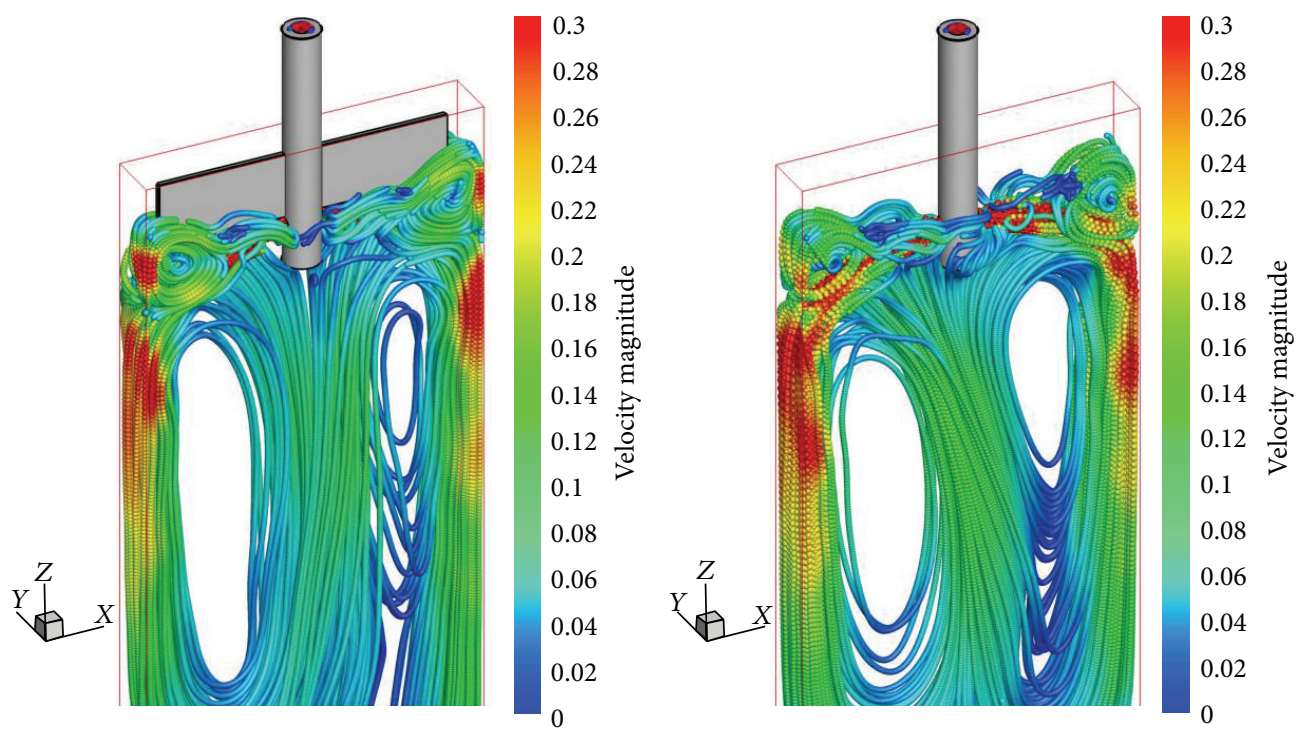

FIgURE 10: The fluid flow pattern inside the mold using the SEN with flow conditioners and a traditional SEN is depicted through stream traces starting at the SEN inlet port.

As expected, the fluid conditioners attached to the SEN increase the average fluid velocity magnitude at the mold upper zone. However, the average $x$-velocity constitutes the $88 \%$ of the average velocity magnitude when using the SEN with flow conditioners. This value contrasts with the corresponding one for a traditional SEN, which is only $72 \%$.

In accordance with several authors, some distortions of the ideal fluid flow pattern in the mold upper zone eventually induce deviations of the ideal pattern in the mold middle zone, and vice versa $[19,20]$. In Figure 10, the fluid flow pattern inside the mold is depicted through stream traces starting at the SEN inlet port. This figure shows the results for both, a traditional SEN and the SEN with flow conditioners.
The number of stream traces for each case is the same. The starting point for each stream trace is the same in both cases. The markers of the stream traces are small spheres colored in accordance with its velocity magnitude. Figure 10 shows that the SEN with fluid flow conditioners improves at the same time the symmetry in the mold upper and middle zones.

Figure 10 also shows that the fluid velocities at the mold narrow walls remain almost unaffected. This result suggests that the SEN with fluid flow conditioners does not negatively alter the formation of the steel solidified shell. Despite this, effect of the fluid flow conditioners on the formation of steel solidified shell must be carefully evaluated before its implementation at industry. 

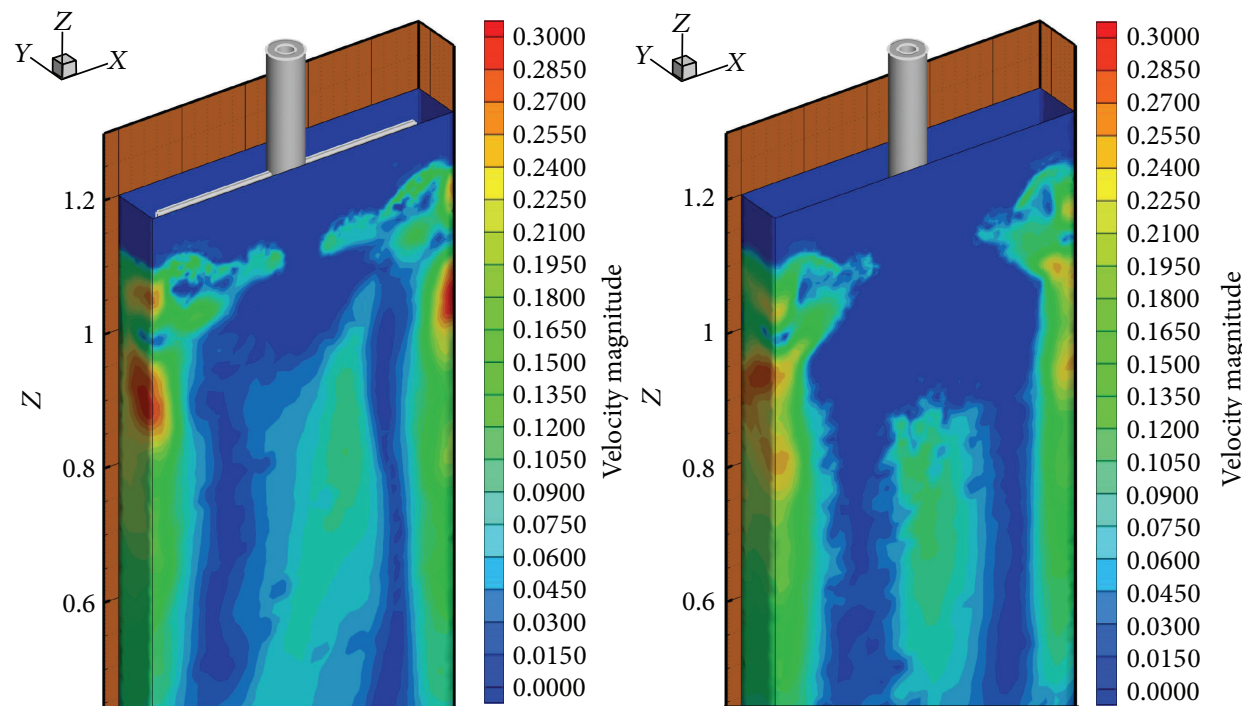

FIGURE 11: Contours map of fluid velocity magnitude at the mold walls using the SEN with fluid flow conditioners and a traditional SEN.

The results of numerical simulations previously presented confirm that the fluid flow pattern inside the mold when using the nozzle with fluid flow conditioners match the expected one. This means that the performance of the proposed SEN is better than that of a traditional nozzle. In addition, the results of physical experiments discussed in the next section also support the findings with numerical simulations.

The relationship between oscillation marks on the slab surface and heat transfer is widely recognized [21]. The effect of the fluid flow pattern on the heat transfer and solidification process at the mold upper zone is characterized in [22]. This work found that when the downward degree of the nozzle port angle is decreased from $30^{\circ}$ to $15^{\circ}$, the thickness of the solidified steel shell at the bottom of the mold decreases to half. Figure 11 shows the liquid velocity magnitude at the mold walls using the SEN with flow conditioners and a traditional SEN. This figure shows that the downward angle of the nozzle jet remains almost unchanged when using the SEN with fluid flow conditioners. The jet impinges at the same height on the mold narrow walls. This figure also shows a slight increase of the velocity at the free surface, near the middle of the mold wide walls. Qualitatively, the formation of the solidified layer is not degraded by using a nozzle with flow modifiers. Nevertheless, further work is necessary to quantitatively evaluate the effect of the fluid flow modifiers.

\section{Physical Evaluation of the SEN with Fluid Flow Conditioners}

The scaled model is a rectangular prism made of acrylic plates. The physical dimensions of the physical model were described in Section 3.3. Further details about the physical model can be found in previous works of the same authors $[12,23]$.

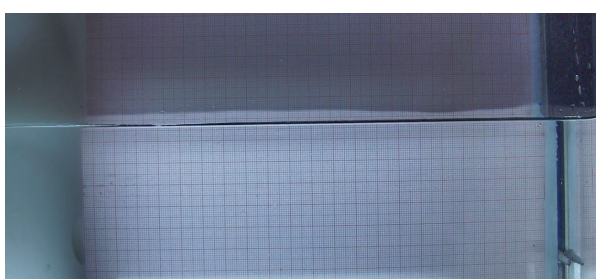

FIGURE 12: Liquid free surface under no flow conditions.

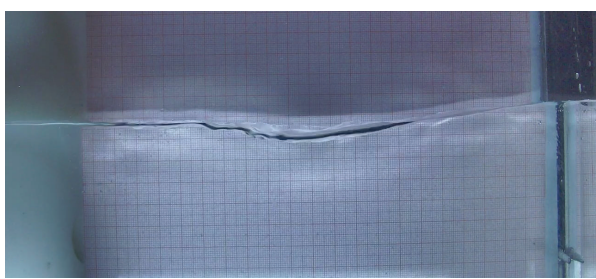

(a)

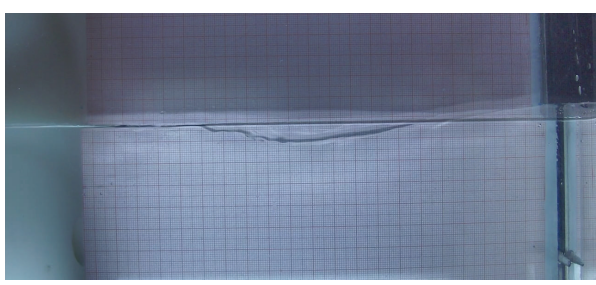

(b)

FIGURE 13: (a) Liquid free surface generated with a traditional SEN. (b) The previous image combined with the reference free surface.

Figure 12 shows the liquid free surface under no flow conditions. This image constitutes the reference value for all the physical experiments of the present work. The back wall of the mold has attached a sheet of graph paper to have a visual reference. All the images from physical experiments 


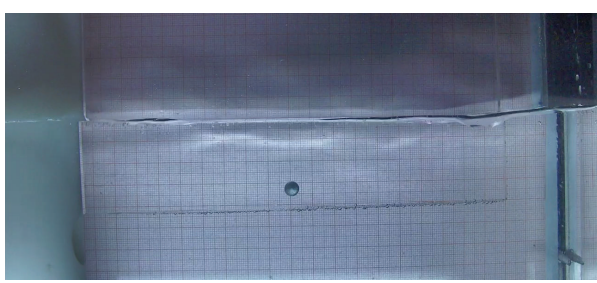

(a)

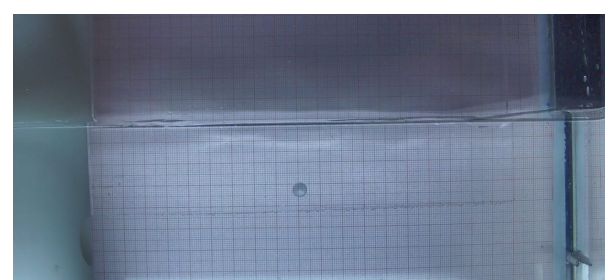

(b)

FIGURE 14: (a) Liquid free surface generated with the SEN with flow conditioners. (b) The previous image combined with the reference free surface.

are snapshots from HD video recordings. To get the best resolution, only a half on the mold upper zone was recorded.

When liquid is flowing through the mold, the free surface of the liquid oscillates permanently. Several authors have argued that the liquid free surface behaves like a standing wave [23]. The frequencies spectra associated with the free surface oscillations have been studied in several works [19, 23]. Figure 13(a) shows a snapshot of the liquid free surface generated with a traditional SEN. This figure reflects the bigger observed distortion of the liquid free surface. Figure 13(a) shows a severely depressed zone at the middle of the distance between the SEN and the mold narrow wall. Note that the shape of the free surface is very similar to that obtained through numerical simulations (see Figure 5). Figure 13(b) combines the images from Figures 12 and 13(a). With respect to the rest condition, the height of the free surface falls nearly $0.008 \mathrm{~m}$ at the trough and rises almost $0.007 \mathrm{~m}$ at the crest.

Figure 14(a) shows the liquid free surface deformation for a SEN with fluid flow conditioners. As in the previous case, this figure reflects the bigger observed distortion of the liquid free surface. Figure 14(b) combines the images from Figures 12 and 14(a). This figure shows that the level of distortion of the free surface is very small. Figure 14(a) shows that the free surface remains flat along almost the entire mold, but rises near the narrow wall of the mold. Again, the shape of the free surface obtained in the physical experiment is very similar to that obtained through numerical simulations (see Figure 5). By comparing Figures 13(b) and 14(b), we can conclude that traditional nozzles produce higher deformations of the free surface.

Figures 15(a) and 15(b) show the fluid flow pattern inside the mold using the nozzle with flow conditioners. In this experiment, small air bubbles allow outlining the flow pattern. The time elapsed between Figures 15(a) and 15(b) is $1 / 12 \mathrm{sec}$. It can be easily observed the double roll pattern. These figures also show an excellent agreement with the liquid velocity fields numerically calculated (see Figure 6).

To test the versatility of the proposed design, Figure 16 shows the results of a variant of the flow conditioners. In this experiment, the fluid flow conditioners are not completely solid. It is observed that these conditioners also decrease the amplitude of variations in the liquid free surface.

Figure 17 show the fluid flow pattern inside the mold using the same variant for the flow conditioners. Again, small air bubbles allow outlining the fluid flow pattern. The time

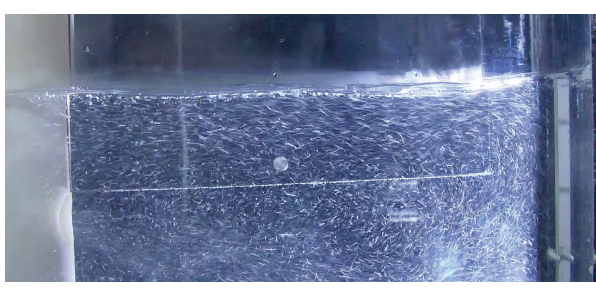

(a)

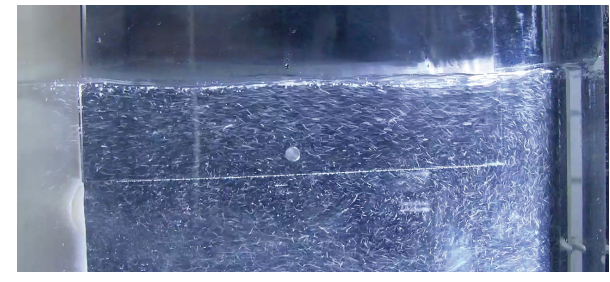

(b)

FIGURE 15: Fluid flow pattern inside the mold using the nozzle with flow conditioners. Small air bubbles outline the flow pattern. The time elapsed between images (a) and (b) is $1 / 12 \mathrm{sec}$.

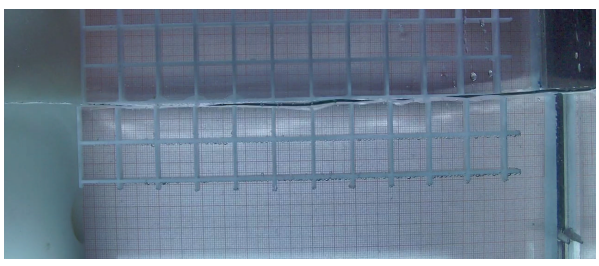

(a)

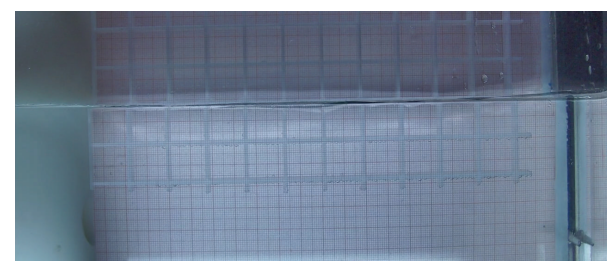

(b)

FIGURE 16: (a) Liquid free surface generated with the SEN with flow conditioners. (b) The previous image combined with the reference free surface. The flow conditioner is a perforated plate instead of the solid one. 


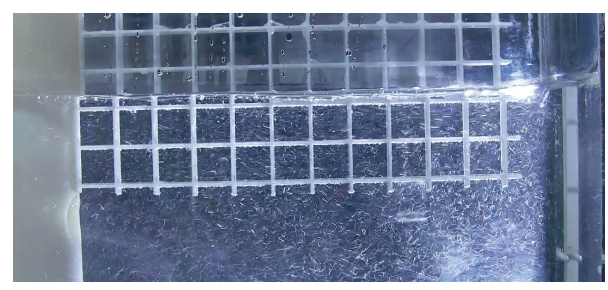

(a)

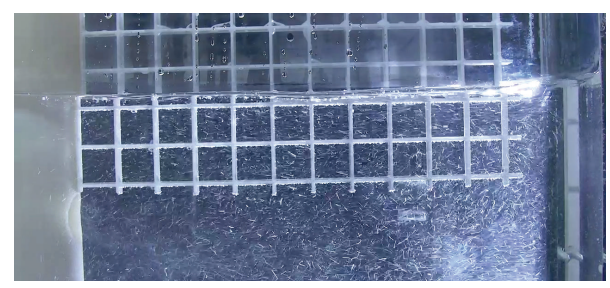

(b)

FIGURE 17: Fluid flow pattern inside the mold using the nozzle with flow conditioners. Small air bubbles outline the flow pattern. The time elapsed between images (a) and (b) is $1 / 12 \mathrm{sec}$. The flow conditioner is a perforated plate instead of the solid one.

elapsed between Figures $17(\mathrm{a})$ and $17(\mathrm{~b})$ is $1 / 12 \mathrm{sec}$. In this case, it is a little more difficult to recognize the double roll fluid flow pattern because the conditioner is not totally translucent. Despite this, a detailed analysis of the whole experiment video shows that the double roll exists.

\section{Concluding Remarks}

Numerical simulations confirm that the nozzle with the fluid flow conditioners produces a fluid flow pattern as expected. At the free surface, the conditioners inhibit the flow from one of the mold wide walls to the other one. Therefore, the occurrence of vortexes at the free surface is minimized.

Numerical simulations also confirmed the anticipated increase of the fluid mean velocity at the mold upper zone. The simulations presented in this work showed that the downward angle of the nozzle jet remains almost unchanged when using the SEN with fluid flow conditioners. Apparently, the formation of the solidified layer is not degraded by using a nozzle with flow modifiers. Nevertheless, the thermal and structural effects of the fluid flow conditioners on the solidification process of the steel inside the mold must be evaluated before its implementation at industry.

Physical simulations probed that the SEN with fluid flow conditioners reduces the fluctuations of the free surface. This work also shows that the fluid flow modifiers may be replaced by a grid that interferes as little as possible to the flux, which serves to insulate the steel and to lubricate the mold.

Some additional features of the proposed design are as follows.

(a) The nozzle with fluid flow conditioners can be easily manufactured using currently available ceramic materials. (b) The shape of the fluid flow conditioners can be easily changed to improve its performance.

(c) The fluid flow conditioners can also be used for cooling purposes by circulating a coolant fluid inside them.

\section{Conflict of Interests}

The authors declare that there is no conflict of interests regarding the publication of this paper.

\section{Acknowledgments}

F. Rivera-Perez (F. R-P) thanks Universidad Autonoma Metropolitana for Ph.D. Grant. R. Hernandez-Santoyo thanks Consejo Nacional de Ciencia y Technología (CONACYT) for Grant no. 284913. This work was done in partial fulfillment of F. R-P's PhD requirements. The numerical and physical simulations were done in the Laboratorio de Computo Cientifico-Departamento de Sistemas at Universidad Autonoma Metropolitana-Azcapotzalco.

\section{References}

[1] Y. J. Jeon, H. J. Sung, and S. Lee, "Flow oscillations and meniscus fluctuations in a funnel-type water mold model," Metallurgical and Materials Transactions B: Process Metallurgy and Materials Processing Science, vol. 41, no. 1, pp. 121-130, 2010.

[2] C. H. Yim and O. Kwon, "Advanced continuous casting technologies for cost reduction and improvement," Journal of Iron and Steel Research International, vol. 15, pp. 52-58, 2008.

[3] B. You, T. Sim, M. Kim, D. Lee, J. Lee, and J. Lee, "Molten steel level control based on an adaptive fuzzy estimator in a continuous caster," ISIJ International, vol. 49, no. 8, pp. 1174$1183,2009$.

[4] V. Singh, S. K. Dash, J. S. Sunitha, S. K. Ajmani, and A. K. Das, "Experimental simulation and mathematical modeling of air bubble movement in slab caster mold," ISIJ International, vol. 46, no. 2, pp. 210-218, 2006.

[5] Q. Yuan, B. G. Thomas, and S. P. Vanka, "Study of transient flow and particle transport in continuous steel caster molds: part II. Particle transport," Metallurgical and Materials Transactions B, vol. 35, no. 4, pp. 703-714, 2004.

[6] Q. Yuan, S. Sivaramakrishnan, S. P. Vanka, and B. G. Thomas, "Computational and experimental study of turbulent flow in a 0.4-scale water model of a continuous steel caster," Metallurgical and Materials Transactions B, vol. 35, no. 5, pp. 967-982, 2004.

[7] L. F. Zhang and B. G. Thomas, "State of the art in evaluation and control of steel cleanliness," ISIJ International, vol. 43, no. 3, pp. 271-291, 2003.

[8] K. Timmel, X. Miao, T. Wondrak et al., "Experimental and numerical modelling of the fluid flow in the continuous casting of steel," The European Physical Journal Special Topics, vol. 220, no. 1, pp. 151-166, 2013.

[9] Y. Chen, L. Zhang, S. Yang, and J. Li, "Water modeling of self-braking submerged entry nozzle used for steel continuous casting mold," JOM, vol. 64, no. 9, pp. 1080-1086, 2012.

[10] C. A. Real-Ramirez and J. I. Gonzalez-Trejo, "Analysis of threedimensional vortexes below the free surface in a continuous 
casting mold," International Journal of Minerals, Metallurgy and Materials, vol. 18, no. 4, pp. 397-407, 2011.

[11] C. Real, R. Miranda, C. Vilchis, M. Barron, L. Hoyos, and J. Gonzalez, "Transient internal flow characterization of a bifurcated submerged entry nozzle," ISIJ International, vol. 46, no. 8, pp. 1183-1191, 2006.

[12] R. Miranda, M. A. Barron, J. Barreto, L. Hoyos, and J. Gonzalez, "Experimental and numerical analysis of the free surface in a water model of a slab continuous casting mold," ISIJ International, vol. 45, no. 11, pp. 1626-1635, 2005.

[13] B. Shen, H. Shen, and B. Liu, "Instability of fluid flow and level fluctuation in continuous thin slab casting mould," ISIJ International, vol. 47, no. 3, pp. 427-432, 2007.

[14] R. D. Dauby and H. Pierre, "Real flows in continuous casting," AIST Transactions, vol. 8, no. 6, pp. 152-160, 2011.

[15] Y. Wang and L. Zhang, "Fluid flow-related transport phenomena in steel slab continuous casting strands under electromagnetic brake," Metallurgical and Materials Transactions B: Process Metallurgy and Materials Processing Science, vol. 42, no. 6, pp. 1319-1351, 2011.

[16] Y. H. Wu and B. Wiwatanapataphee, "Modelling of turbulent flow and multi-phase heat transfer under electromagnetic force," Discrete and Continuous Dynamical Systems B: A Journal Bridging Mathematics and Sciences, vol. 8, no. 3, pp. 695-706, 2007.

[17] M. Kamal and Y. Sahai, "A simple innovation in continuous casting mold technology for fluid flow and surface standing waves control," ISIJ International, vol. 46, no. 12, pp. 1823-1832, 2006.

[18] ANSYS, ANSYS FLUENT Theory Guide, ANSYS, Canonsburg, $\mathrm{Pa}, \mathrm{USA}, 2012$.

[19] D. Gupta, S. Chakraborty, and A. K. Lahiri, "Asymmetry and oscillation of the fluid flow pattern in a continuous casting mould: a water model study," ISIJ International, vol. 37, no. 7, pp. 654-658, 1997.

[20] D. Gupta and A. K. Lahiri, "Water-modeling study of the surface disturbances in continuous slab caster," Metallurgical and Materials Transactions B, vol. 25, no. 2, pp. 227-233, 1994.

[21] J. M. Hill, Y. H. Wu, and B. Wiwatanapataphee, "Analysis of flux flow and the formation of oscillation marks in the continuous caster," Journal of Engineering Mathematics, vol. 36, no. 4, pp. 311-326, 1999.

[22] Y. H. Wu, B. Wiwatanapataphee, and X. Yu, "An enthalpy control volume method for transient mass and heat transport with solidification," International Journal of Computational Fluid Dynamics, vol. 18, no. 7, pp. 577-584, 2004.

[23] J. R. Miranda-Tello, F. Sánchez-Rangel, C. A. Real-Ramírez et al., "Characterization of the level fluctuations in a physical model of the steel continuous casting mold through image processing," Revista Mexicana de Fisica, vol. 58, no. 2, pp. 166173, 2012. 


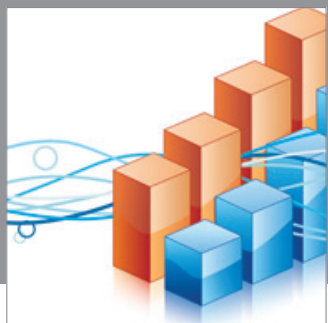

Advances in

Operations Research

mansans

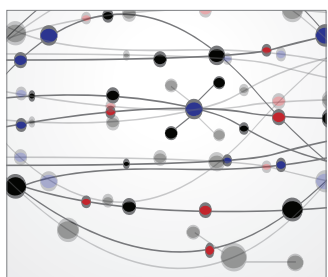

The Scientific World Journal
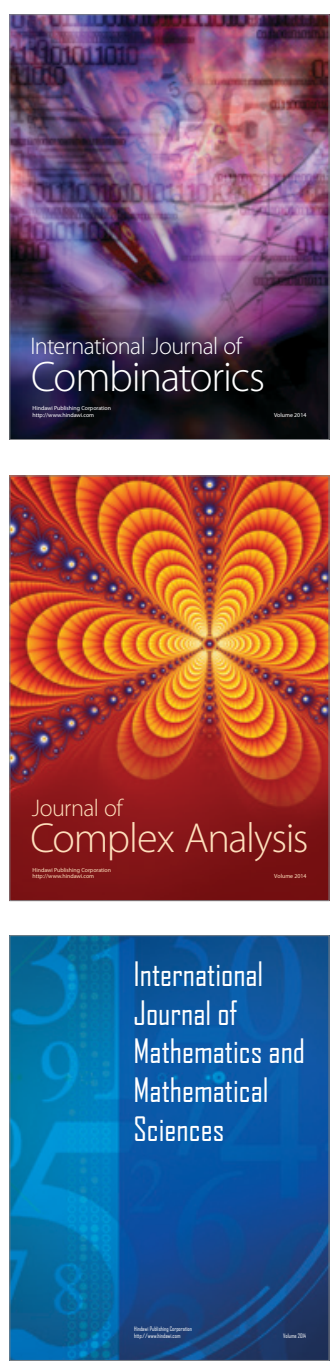
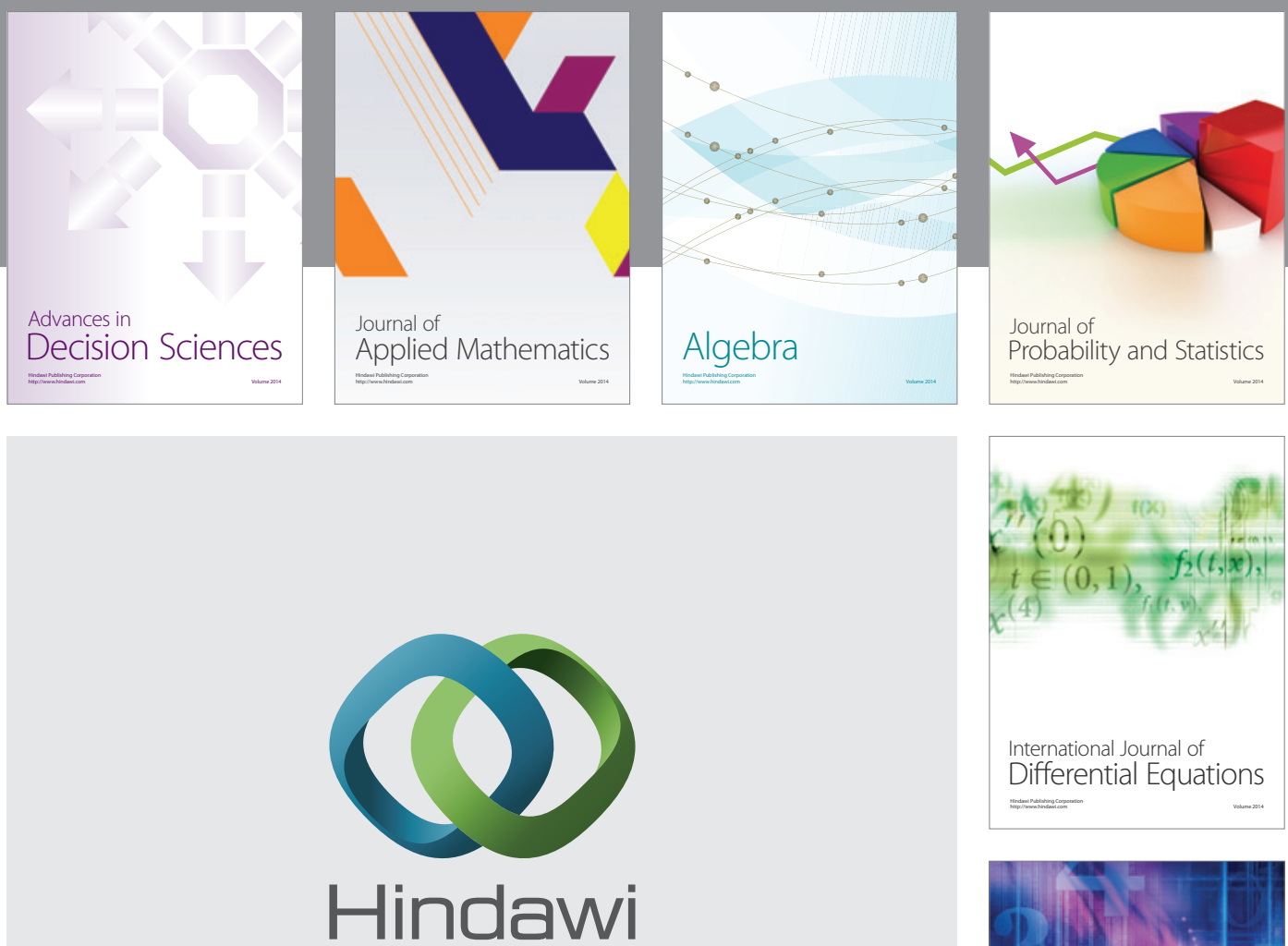

Submit your manuscripts at http://www.hindawi.com
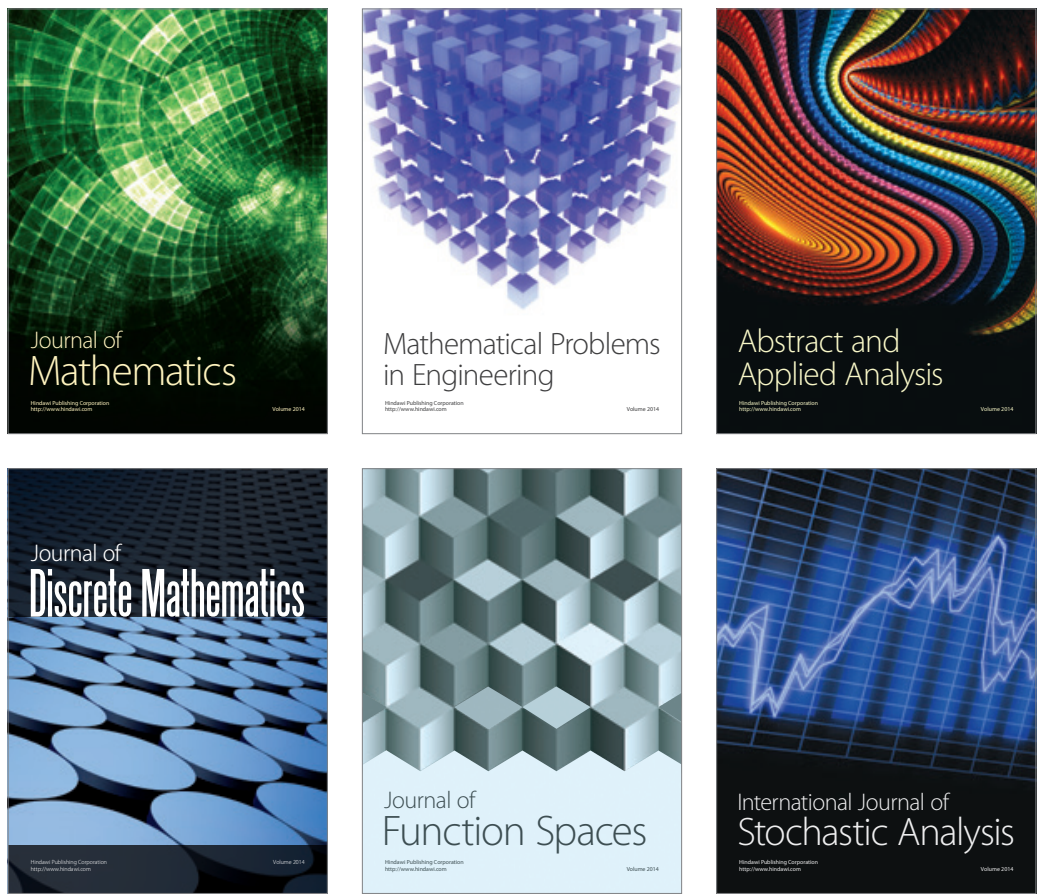

Journal of

Function Spaces

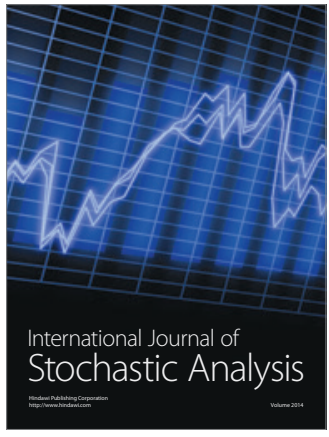

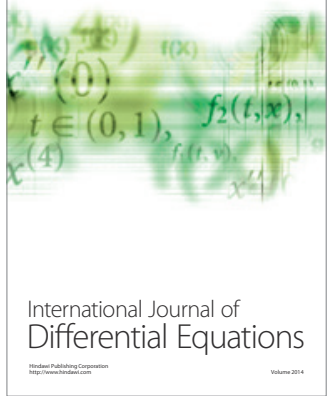
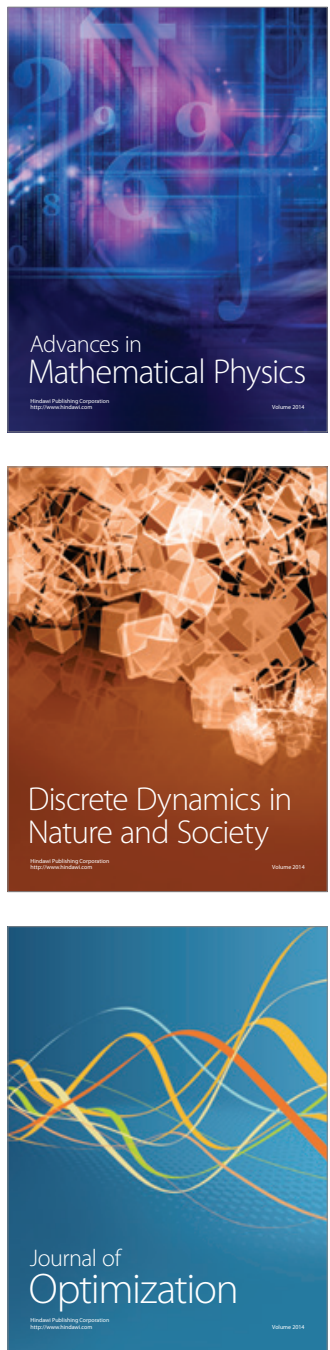